\title{
On character amenability of semigroup algebras
}

\author{
S.M. Maepa \\ Department of Mathematics and Applied Mathematics, University of Pretoria, Pretoria, \\ South Africa. \\ E-Mail charles.maepa@up.ac.za \\ O. T. Mewomo \\ School of Mathematics, Statistics and Computer Sciences, University of Kwazulu-Natal, \\ Durban, South Africa. \\ E-Mail mewomoo@ukzn.ac.za
}

\begin{abstract}
We study the character amenability of semigroup algebras. We work on general semigroups and certain semigroups such as inverse semigroups with a finite number of idempotents, inverse semigroups with uniformly locally finite idempotent set, Brandt and Rees semigroup and study the character amenability of the semigroup algebra $l^{1}(S)$ in relation to the structures of the semigroup $S$. In particular, we show that for any semigroup $S$, if $\ell^{1}(S)$ is character amenable, then $S$ is amenable and regular. We also show that the left character amenability of the semigroup algebra $\ell^{1}(S)$ on a Brandt semigroup $S$ over a group $G$ with index set $J$ is equivalent to the amenability of $G$ and $J$ being finite. Finally, we show that for a Rees semigroup $S$ with a zero over the group $G$, the left character amenability of $\ell^{1}(S)$ is equivalent to its amenability, this is in turn equivalent to $G$ being amenable.
\end{abstract}

\section{Introduction}

The notion of amenability in Banach algebra was initiated by Johnson in [16]. Since then, a substantial amount of research has been done to link amenability and other notions of amenability to various properties of Banach algebras, see [19].

${ }^{1} 2000$ Mathematics Subject Classification: Primary: 46H20. Secondary: 46H10, 46H25 Key Words: Semigroup, Semigroup algebra, Banach algebra, character, left character amenable, character amenable. 
Let $A$ be a Banach algebra over $\mathbb{C}$ and $\varphi: A \rightarrow \mathbb{C}$ be a character on $A$, that is, an algebra homomorphism from $A$ into $\mathbb{C}$, and let $\Phi_{A}$ denote the character space of $A$ (that is, the set of all characters on $A$ ). In [22], see also [15], Monfared introduced the notion of character amenable Banach algebras. His definition of this notion requires continuous derivations from $A$ into dual Banach $A$-bimodules to be inner, but only those modules are concerned where either of the left or right module action is defined by characters on A. As such character amenability is weaker than the classical amenability introduced by Johnson in [16], so all amenable Banach algebras are character amenable.

Approximate version of character amenability for Banach algebras was first introduced by Aghababa, Shi and Wu in [1] and further studied by the first author and Okelo in [21] for algebras defined over locally compact groups and second dual of Banach algebra.

Module character amenability of Banach algebras which defines the notion of invariant functional with respect to a Banach Bimodule with compatible actions and application to the semigroup algebras of inverse semigroup is also introduced by the authors in [3].

Various aspects of cohomologies of semigroup algebras have been studied by several authors, most notably are Duncan and Namioka [9], Duncan and Paterson [10], Gronbaek [12], Sadr and Pourabbas [24], Lashkarizadeh and Samea [6], Mewomo [18], Mewomo and Ogunsola [20] and Dales, Lau and Strauss [8].

It is shown in [9] that the amenability of semigroup algebra $\ell^{1}(S)$ implies that the semigroup $S$ is amenable. The authors in [24] characterized the approximate amenability of Brandt semigroup algebras. In particular, they showed that for a Brandt semigroup $S$ over a group $G$ with nonempty index set $J$, the semigroup algebra $\ell^{1}(S)$ is approximately amenable if and only if $G$ is amenable and $J$ is finite.

No much work has been done to date on the character amenability version for semigroup algebra $\ell^{1}(S)$ on a semigroup $S$, as in the other notions of amenability. It will be good to study this and see how the character amenability of $\ell^{1}(S)$ affects the structure of $S$. Thus, in this work, we study the character 
amenability of semigroup algebras. We focus on certain semigroups such as inverse semigroup with a finite number of idempotents, inverse semigroup with uniformly locally finite idempotent set, Rees Semigroup, Clifford semigroup and Brandt semigroup and study the character amenability of $\ell^{1}(S)$ in relation to the semigroup $S$.

\section{Preliminaries}

First, we recall some standard notions; for further details, see [7], [8] and [19].

Let $A$ be an algebra. The character space of $A$ is denoted by $\Phi_{A}$. Let $X$ be an $A$-bimodule. A derivation from $A$ to $X$ is a linear map $D: A \rightarrow X$ such that

$$
D(a b)=D(a) \cdot b+a \cdot D(b) \quad(a, b \in A) .
$$

For example, for $x \in X$, the map $\delta_{x}: A \rightarrow X$ defined by

$\delta_{x}(a)=a \cdot x-x \cdot a \quad(a \in A)$ is a derivation; derivations of this form are called the inner derivations.

Let $A$ be a Banach algebra, and let $X$ be an $A$-bimodule. Then $X$ is a Banach $A$-bimodule if $X$ is a Banach space and if there is a constant $k>0$ such that

$$
\|a \cdot x\| \leq k\|a\|\|x\|, \quad\|x \cdot a\| \leq k\|a\|\|x\| \quad(a \in A, x \in X) .
$$

By renorming $X$, we can suppose that $k=1$. For example, $A$ itself is Banach $A$-bimodule, and $X^{\prime}$, the dual space of a Banach $A$-bimodule $X$, is a Banach $A$-bimodule with respect to the module operations specified for by

$$
\langle x, a \cdot \lambda\rangle=\langle x \cdot a, \lambda\rangle, \quad\langle x, \lambda \cdot a\rangle=\langle a \cdot x, \lambda\rangle \quad(x \in X)
$$

for $a \in A$ and $\lambda \in X^{\prime}$; we say that $X^{\prime}$ is the dual module of $X$.

The Banach algebra $A$ is amenable if every continuous derivation $D: A \rightarrow X^{\prime}$ is inner for each Banach $A$-bimodule $X$.

We let $\mathcal{M}_{\varphi_{r}}^{A}$ denote the class of Banach $A$ - bimodule $X$ for which the right module action of $A$ on $X$ is given by $x \cdot a=\varphi(a) x \quad\left(a \in A, x \in X, \varphi \in \Phi_{A}\right)$, 
and $\mathcal{M}_{\varphi_{l}}^{A}$ denote the class of Banach $A$ - bimodule $X$ for which the left module action of $A$ on $X$ is given by $a \cdot x=\varphi(a) x \quad\left(a \in A, x \in X, \varphi \in \Phi_{A}\right)$. If the right module action of $A$ on $X$ is given by $x \cdot a=\varphi(a) x$, then it is easy to see that the left module action of $A$ on the dual module $X^{\prime}$ is given by $a \cdot f=\varphi(a) f \quad\left(a \in A, f \in X^{\prime}, \varphi \in \Phi_{A}\right)$. Thus, we note that $X \in \mathcal{M}_{\varphi_{r}}^{A}$ (resp. $X \in \mathcal{M}_{\varphi_{l}}^{A}$ ) if and only if $X^{\prime} \in \mathcal{M}_{\varphi_{l}}^{A}$ (resp. $X^{\prime} \in \mathcal{M}_{\varphi_{r}}^{A}$ ).

Let $A$ be a Banach algebra and let $\varphi \in \Phi_{A}$, we recall from [15], see also [22] that

(i) $A$ is left $\varphi$-amenable if every continuous derivation $D: A \rightarrow X^{\prime}$ is inner for every $X \in \mathcal{M}_{\varphi_{r}}^{A}$;

(ii) $A$ is right $\varphi$-amenable if every continuous derivation $D: A \rightarrow X^{\prime}$ is inner for every $X \in \mathcal{M}_{\varphi_{l}}^{A}$;

(iii) $A$ is left character amenable if it is left $\varphi$-amenable for every $\varphi \in \Phi_{A}$;

(iv) $A$ is right character amenable if it is right $\varphi$-amenable for every $\varphi \in \Phi_{A}$;

(v) $A$ is character amenable if it is both left and right character amenable.

We also recall from [15] that, for $\varphi \in \Phi_{A}$, a left (right) $\varphi$-virtual diagonal for $A$ is an element $M$ in $(A \hat{\otimes} A)^{\prime \prime}$ such that

$$
\text { (i) } M \cdot a=\varphi(a) M \quad(a \cdot M=\varphi(a) M) \quad(a \in A) \text {; }
$$

(ii) $\langle M, \varphi \otimes \varphi\rangle=\pi^{\prime \prime}(M)(\varphi)=1$,

where $\pi: A \hat{\otimes} A \rightarrow A$ defined by $\pi(a \otimes b)=a b \quad(a, b \in A)$ is the product map.

It was shown in [15, Theorem 2.3] that a Banach algebra $A$ is left [right] $\varphi$-amenable for $\varphi$ in $\Phi_{A}$ if and only if it has a left [right] $\varphi$-virtual diagonal. This characterization will be used in the work.

We recall that a semigroup is a non-empty set $S$ with an associative binary operation

$$
(s, t) \rightarrow s t ; \quad S \times S \rightarrow S \quad(s, t \in S) .
$$

Let $S$ be a semigroup, $S$ is said to be regular if for all $s \in S$, there is $s^{*} \in S$ such that $s s^{*} s=s$ and $s^{*} s s^{*}=s^{*} . S$ is an inverse semigroup if such $s^{*}$ exists and is unique for all $s \in S$. An element $p \in S$ is idempotent if $p^{2}=p$. The set 
of idempotents in $S$ is denoted by $E(S)$. A semigroup $S$ is semilattice if $S$ is commutative and $E(S)=S$.

Let $S$ be a non-empty set. Then

$$
\ell^{1}(S)=\left\{f \in \mathbb{C}^{S}: \sum_{s \in S}|f(s)|<\infty\right\}
$$

with the norm $\|\cdot\|_{1}$ given by $\|f\|_{1}=\sum_{s \in S}|f(s)|$ for $f \in \ell^{1}(S)$. We write $\delta_{s}$ for the characteristic function of $\{s\}$ when $s \in S$.

Now suppose that $S$ is a semigroup. For $f, g \in \ell^{1}(S)$, we set

$$
(f \star g)(t)=\left\{\sum f(r) g(s): r, s \in S, r s=t\right\} \quad(t \in S)
$$

so that $f \star g \in \ell^{1}(S)$. It is standard that $\left(\ell^{1}(S), \star\right)$ is a Banach algebra, called the semigroup algebra on $S$. For a further discussion of this algebra, see $[7,8]$. In particular, with $A=\ell^{1}(S)$, we identify $A^{\prime}$ with $C(\beta S)$, where $\beta S$ is the Stone-Čech compactification of $S$, and $\left(A^{\prime \prime}, \square\right)$ with $(M(\beta S), \square)$, where $M(\beta S)$ is the space of regular Borel measures on $\beta S$ of $S$; in this way, $(\beta S, \square)$ is a compact, right topological semigroup that is a subsemigroup of $(M(\beta S), \square)$ after the identification of $u \in \beta S$ with $\delta_{u} \in M(\beta S) \cdot \ell^{1}(S)$ is commutative if and only if $S$ is abelian.

There is always one character on the Banach algebra $\ell^{1}(S)$ : this is the augmentation character

$$
\varphi_{S}: f \mapsto \sum_{s \in S} f(s), \quad l^{1}(S) \rightarrow \mathbb{C} .
$$

Let $T$ be a subsemigroup of a semigroup $S$. Then

$$
\Phi_{\ell^{1}(T)}=\left\{\varphi_{S} \mid \ell^{1}(T): \varphi_{S} \in \Phi_{\ell^{1}(S)}\right\} .
$$

\section{General Results}

In this section, we prove some general results which are useful in establishing our main results on semigroup algebras. 
We note that the concept of $\varphi$-amenable Banach algebra in [17] is the same as right $\varphi$-amenable in [22], see also [17, Theorem 1.1]. Also, any statement about right $\varphi$-amenability turns into an analogous statement about left $\varphi$-amenability.

Proposition 3.1 Let $A$ be a left character amenable Banach algebra and I a closed ideal of $A$. Then $A / I$ is left character amenable.

Proof This follows from the fact that $\tau: A \rightarrow A / I$ is a continuous homomorphism with dense range and [22, Theorem 2.6].

Let $A \hat{\otimes} B$ be the projective tensor product of Banach algebras $A$ and $B$. For $f \in A^{\prime}, g \in B^{\prime}$, let $f \otimes g \in(A \hat{\otimes} B)^{\prime}$ such that

$$
(f \otimes g)(a \otimes b)=f(a) g(b) \quad(a \in A, b \in B) .
$$

Then $\Phi_{A \hat{\otimes} B}=\left\{\varphi \otimes \psi: \varphi \in \Phi_{A}, \psi \in \Phi_{B}\right\}$. The following result is from [17]:

Proposition 3.2 Let $A$ and $B$ be Banach algebras with $\varphi \in \Phi_{A}$ and $\psi \in \Phi_{B}$. Then $A \hat{\otimes} B$ is $(\varphi \otimes \psi)$-amenable if and only if $A$ is $\varphi$-amenable and $B$ is $\psi$ amenable. In particular, $A \hat{\otimes} B$ is left character amenable if and only if $A$ and $B$ are left character amenable.

Let $A$ be a Banach algebra and let $J$ be a non-empty set. We denote by $\mathcal{M}_{J}(A)$ the set of $J \times J$ matrices $\left(a_{i j}\right)$ with entries in $A$ such that

$$
\left\|\left(a_{i j}\right)\right\|=\sum_{i, j \in J}\left\|a_{i j}\right\|<\infty .
$$

Then $\mathcal{M}_{J}(A)$ with the usual matrix multiplication is a Banach algebra that belongs to the class of $l^{1}$-Munn algebras introduced in [11]. The map $\tau: \mathcal{M}_{J}(A) \rightarrow A \hat{\otimes} \mathcal{M}_{J}(\mathbb{C})$ defined by $\tau\left(\left(a_{i j}\right)\right)=\sum_{i, j \in J} a_{i j} \otimes E_{i j}$ $\left(\left(a_{i j}\right) \in \mathcal{M}_{J}(A)\right)$, is an isometric isomorphism of Banach algebras, where $\left(E_{i j}\right)$ are the matrix units in $\mathcal{M}_{J}(\mathbb{C})$. With this identification and Proposition 3.2, we have the next result.

Corollary 3.3 Let $A$ be a Banach algebra and $J$ a non-empty set. Then $\mathcal{M}_{J}(A)$ is left character amenable if and only if $A$ is left character amenable. 
Proposition 3.4 Let $G$ be a group and $n \in \mathbb{N}$. Then $\mathcal{M}_{n}\left(\ell^{1}(G)\right)$ is left character amenable if and only if it is amenable.

Proof Suppose $\mathcal{M}_{n}\left(\ell^{1}(G)\right)$ is left character amenable, then $\ell^{1}(G)$ is left character amenable by Corollary 3.3. Also, using [22, Corollary 2.4], $\ell^{1}(G)$ is left character amenable if and only $G$ is amenable and $G$ is amenable by the classical result of Johnson in [16] if and only if $\ell^{1}(G)$ is amenable. Finally, by [8, Theorem 2.7(i)], $\mathcal{M}_{n}\left(\ell^{1}(G)\right)$ is amenable.

Recently, Amini, Essmaili and Rostami in [2] introduced the notion of left [right] $\phi$-biflatness for a Banach algebra $A$ and $\varphi \in \Phi_{A}$. We recall from [2] that a Banach algebra $A$ is left [right] $\varphi$-biflat if there exists a bounded linear operator $\rho: A \rightarrow(A \hat{\otimes} A)^{\prime \prime}$ such that

$$
\begin{gathered}
\text { (i) } \rho(a b)=\varphi(a) \rho(b)=\rho(a) * b \quad[\rho(a b)=\varphi(b) \rho(a)=a \cdot \rho(b)] \\
(i i) \quad\left(\pi_{A}^{\prime \prime} \circ \rho(a)\right)(\varphi)=\varphi(a) \quad\left(a, b \in A, \varphi \in \Phi_{A}\right) .
\end{gathered}
$$

It was remarked in [2], that $\varphi$-biflatness of $A$ in the sense of Sahami and Pourabbas in [25] implies left $\varphi$-biflatness in the sense of [2].

Helemskii in [13] showed that a Banach algebra $A$ is amenable if and only if it is biflat and has a bounded approximate identity. We give the character amenability version of this result.

The next result is [2, Proposition 2.2].

Proposition 3.5 Let $A$ be a Banach algebra with $\varphi \in \Phi_{A}$.

(i) If $A$ is left $\varphi$-amenable, then $A$ is left $\varphi$-biflat;

(ii If $A$ is left $\varphi$-biflat and has a bounded approximate identity, then $A$ is left $\varphi$-amenable.

The definition of $\varphi$-biflatness in [2] can be generalized as follows:

We say that a Banach algebra $A$ is

(i) left [right] character biflat if it is left [right] $\varphi$-biflat for every $\varphi \in \Phi_{A}$;

(ii) character biflat if it is both left and right character biflat. 
Theorem 3.6 Let $A$ be a Banach algebra. Then the following are equivalent:

(i) $A$ is character amenable

(ii) A is character biflat and has a bounded approximate identity.

Proof This clearly follows from Proposition 3.5 (i) and (ii) and the above definition.

\section{Results on semigroup algebras}

In this section, we shall consider the character amenability properties of semigroup algebras.

Let $S$ be a semigroup, for each $\varphi \in \Phi_{S}$, the map $\tilde{\varphi}: \ell^{1}(S): \mathbb{C}$ defined by

$$
\tilde{\varphi}\left(\sum_{s \in S} \alpha_{s} \delta_{s}\right)=\sum_{s \in S} \alpha_{s} \varphi(s)
$$

is a character on $\ell^{1}(S)$, and every character on $\ell^{1}(S)$ arises in this way. Also, we often regard an element $s \in S$ as an element of $\ell^{1}(S)$, See [8] for details.

For a semigroup $S, \ell^{1}(S) \hat{\otimes} \ell^{1}(S)$ is isometrically isomorphic to $\ell^{1}(S \times S)$, and so, we identify $\left(\ell^{1}(S) \hat{\otimes} \ell^{1}(S)\right)^{\prime \prime}$ with $\ell^{1}(S \times S)^{\prime \prime}$. Under this identification, the bimodule operations are defined as follows: Let $M \in\left(\ell^{\infty}(S \times S)\right)^{\prime}, s \in S$. Then for all $f \in \ell^{\infty}(S \times S)$,

$$
M s(f)=M(s f), \quad s M(f)=M(f s),
$$

where

$$
f s(u, v)=f(s u, v), \quad s f(u, v)=f(u, v s) .
$$

Also, for all $\Psi \in \ell^{\infty}(S)$, we have

$$
\pi^{\prime \prime} M(\Psi)=M\left(\pi^{\prime} \Psi\right), \quad \text { where } \pi^{\prime} \Psi(u, v)=\Psi(u v) \quad(u, v \in S) .
$$

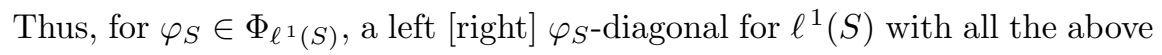
identification and explanation, can be defined as follows:

An element $M \in \ell^{\infty}(S \times S)^{\prime}$ is a 
(i) left $\varphi_{S}$-virtual diagonal for $\ell^{1}(S)$ if

$$
M s=\varphi_{S}(s) M \quad \text { and }\left\langle M, \varphi_{S} \otimes \varphi_{S}\right\rangle=\left(\pi^{\prime \prime} M\right) s\left(\varphi_{S}\right)=1
$$

(ii) right $\varphi_{S}$-virtual diagonal for $\ell^{1}(S)$ if

$$
s M=\varphi_{S}(s) M \quad \text { and }\left\langle M, \varphi_{S} \otimes \varphi_{S}\right\rangle=\left(\pi^{\prime \prime} M\right) s\left(\varphi_{S}\right)=1 ;
$$

(iii) $\varphi_{S}$-virtual diagonal for $\ell^{1}(S)$ if it is both a left $\varphi_{S}$-virtual diagonal and right $\varphi_{S}$-virtual diagonal for $\ell^{1}(S)$.

For a semigroup $S$, given $f \in \ell^{\infty}(S), t \in S$, we define the translation functions $f t, t f$ by

$$
f t(s)=f(t s), \quad t f(s)=f(s t) \quad(s, t \in S) .
$$

A continuous linear function $\mu$ on $\ell^{\infty}(S)$ is called

(i) a mean if $\mu(\mathbf{1})=\|\mu\|=1$, where $\mathbf{1}$ denotes the constant unit function on $S$;

(ii) a left invariant if for each $f \in \ell^{\infty}(S), t \in S$, we have $\mu(f t)=\mu(f)$;

(iii) a right invariant if for each $f \in \ell^{\infty}(S), t \in S$, we have $\mu(t f)=\mu(f)$.

The semigroup $S$ is left [right] amenable if there exists a left [right] invariant mean on $\ell^{\infty}(S)$, and it is amenable if it is left and right amenable. For an inverse semigroup, left amenability is equivalent to right amenability, in which case, amenability is equivalent to left or right amenability. For detail see [9].

Proposition 4.1 Let $S$ be a semigroup and $\varphi_{S} \in \Phi_{\ell^{1}(S)}$.

(i) If $\ell^{1}(S)$ is left [right] $\varphi_{S}$-amenable, then $S$ is left [right] amenable.

(ii) If $\ell^{1}(S)$ is character amenable, then $S$ is amenable and regular.

Proof (i) Since $\ell^{1}(S)$ is left [right] $\varphi_{S}$-amenable, then by [15, Theorem 2.3], it has a left [right] $\varphi_{S}$-virtual diagonal. The rest of the proof follows by simply using the left [right] $\varphi_{S^{-}}$virtual diagonal for $\ell^{1}(S)$ described above and a simple modification of the argument used in the proof of [9, Lemma 3], to obtain a left [right] invariant mean for $S$. 
(ii) That $S$ is amenable clearly follows from (i) and the fact that $\ell^{1}(S)$ is character amenable implies it is both left and right character amenable. Also, by using similar argument in [10, Theorem 1], we show that for each $u \in S$, $s S \cap\left[u u^{-1}\right] \neq \emptyset$, where $\left[u u^{-1}\right]=\{x \in S: x u=u\}$ and this shows that $S$ is regular using [10, Corollary 2].

Corollary 4.2 Let $S$ be a semigroup with $E(S)$ finite. If $\ell^{1}(S)$ is character amenable, then it has an identity.

Proof Since $\ell^{1}(S)$ is character amenable, then it has a left approximate identity and a right approximate identity by [22, Theorem 2.3]. Also, $S$ is regular by Proposition 4.1(ii). Since $E(S)$ is assumed to be finite, then $\ell^{1}(S)$ has an identity using [8, Proposition 4.3].

Remark Proposition 4.1 (i) is true for any semigroup, but the converse is false. The partial converse is given below.

Proposition 4.3 Let $S$ be a finite inverse semigroup and $\varphi_{S} \in \Phi_{\ell^{1}(S)}$. Suppose $S$ is left amenable, then $\ell^{1}(S)$ is left $\varphi_{S}$-amenable.

Proof Since $S$ is finite and inverse, then $\ell^{1}(S)$ is semisimple and finite dimensional by $\left[4\right.$, Theorem 5.2.6], and so $\ell^{1}(S)$ is left $\varphi_{S^{-}}$-amenable.

We recall that a Brandt semigroup $S$ over a group $G$ with index set $J$ is the semigroup consisting of elementary $J \times J$ matrices over $G \cup\{0\}$ and a zero matrix $\mathbf{0}$. We write $S=\left\{(g)_{i j}: g \in G, i, j \in J\right\} \cup\{\mathbf{0}\}$, with multiplication given by

$$
(g)_{i j}(h)_{k l}=\left\{\begin{array}{cc}
(g h)_{i l} & \text { if } j=k \\
\mathbf{0} & \text { if } j \neq k
\end{array}\right.
$$

The Brandt semigroup is an inverse semigroup.

Proposition 4.4 Let $S$ be a Brandt semigroup over a group $G$ with finite index set J. Suppose $G$ is amenable, then $\ell^{1}(S)$ is left character amenable. 
Proof Let $S_{1}$ be a Brandt semigroup over the trivial group with index set $J$. Then $\ell^{1}\left(S_{1}\right)$ is semisimple and finite dimensional, and so is left character amenable. Since $G$ is amenable, then $\ell^{1}(G)$ is left character amenable by $[22$, Corollary 2.4], and so $\ell^{1}(G) \hat{\otimes} \ell^{1}\left(S_{1}\right)$ is left character amenable by Proposition 3.2. But $\ell^{1}(S)$ may be identified with $\ell^{1}\left(S_{1} \times G\right) / \ell^{1}(T)$, where $T$ is the ideal $\{(\mathbf{0}, g): g \in G\}$ of $S_{1} \times G$. Thus $\ell^{1}(S)$ is left character amenable by Proposition 3.1

Remark It was pointed to the authors by the referee that a generalization of Proposition 4.4 for Brandt semigroups is already obtained by Essmaili and Filali in [5, Corrollry 2.7]. Our method and approach is completed different from those used in [5].

Theorem 4.5 Let $S$ be an inverse semigroup with $E(S)$ finite. Then $\ell^{1}(S)$ is left character amenable if each maximal subgroup of $S$ is amenable.

Proof Since $E(S)$ is finite and $S$ is inverse, then $S$ has a principal series

$$
S=I_{1} \supset I_{2} \supset I_{3} \supset \ldots \supset I_{m-1} \supset I_{m}=K(S)
$$

of ideals of $S$, where $K(S)$ is the minimum ideal, see [8, Theorem 3.12]. $I_{j} / I_{j+1}$ is a simple inverse semigroup with a finite number of idempotents, and so is a group. Also, for $i=1,2, \ldots, n-1, I_{i} / I_{i+1}$ is 0 -simple with a finite number of idempotents, and so is a completely 0-simple inverse semigroup, that is a Brandt semigroup. Thus, by Proposition 4.4 and Proposition $3.1, \ell^{1}(S)$ is left character amenable if and only if $\ell^{1}\left(S_{i} / S_{i+1}\right)$ is left character amenable for $i=1,2, . ., n$. For $i=1,2, \ldots, n-1$, let $G_{i}$ be the group of the Brandt semigroup $S_{i} / S_{i+1}$ and let $G_{n}$ be the group $S_{n} / S_{n+1}$. Then $\ell^{1}\left(S_{i} / S_{i+1}\right)$ is amenable for $i=1,2, \ldots, n$ if $G_{i}$ is amenable for $i=1,2, \ldots, n$. But the groups $G_{i}$ are maximal subgroups of $S$.

For an inverse Semigroup $S, \ell^{1}(S)$ has a bounded left approximate identity if and only if it has a bounded right approximate identity and hence a bounded approximate identity. 
Proposition 4.6 Let $S$ be a Brandt semigroup with an infinite index set over an arbitrary group. Then $\ell^{1}(S)$ is not left character amenable.

Proof For a Brandt semigroup $S$ with an infinite index set, $\ell^{1}(S)$ does not have a left bounded approximate identity by [9, Corollary 17]. Thus it is not left character amenable by [22, Theorem 2.3].

For an inverse semigroup $S$, and $p \in E(S)$, we set

$$
G_{p}=\left\{s \in S: s s^{-1}=s^{-1} s=p\right\} .
$$

Then $G_{p}$ is a group with identity $p$. It is called the maximal subgroup of $S$ at $p$. We recall that a Clifford semigroup is an inverse semigroup $S$ for which $s s^{-1}=s^{-1} s \quad(s \in S)$. For a Clifford semigroup $S$, we have $s \in G_{s s^{-1}}$, and so $S$ is a disjoint union of the groups $G_{p} \quad(p \in E(S))$. See [14] for more detail.

Corollary 4.7 Let $S$ be a Clifford semigroup over a finite semilattice $Y$ (i.e. an inverse semigroup which is a union of groups, see [4, pp. 127, 128]). Then $\ell^{1}(S)$ is left character amenable if $G_{\alpha}$ is amenable for each $\alpha \in Y$.

Proof This follows from Theorem 4.5.

Let $P$ be a partially ordered set. For $p \in P$, we define

$$
(p]=\{x: x \leq p\} \text { and }[p)=\{x: p \leq x\} .
$$

Then $P$ is locally finite if $(p]$ is finite for each $p \in P$, and is locally $C$ - finite for some constant $C>1$ if $|(p]|<C$ for each $p \in P$. A partially ordered set that is $C$ - finite for some $C$ is uniformly locally finite.

Let $S$ be an inverse semigroup. Then $S$ is [locally finite/ C-locally finite/ uniformly locally finite] if the partially ordered set $(E(S), \leq)$ has the corresponding property.For detail, see [23].

Theorem 4.8 Let $S$ be an inverse semigroup such that $(E(S), \leq)$ is uniformly locally finite. Suppose $\ell^{1}(S)$ is left character amenable. Then each maximal subgroup of $S$ is amenable. 
Proof Since $(E(S), \leq)$ is uniformly locally finite, then $(S, \leq)$ is uniformly locally finite by [23, Proposition 2.4] and so using [23, Theorem 2.18], we have

$$
\ell^{1}(S) \cong l^{1}-\bigoplus\left\{\mathbb{M}_{E\left(D_{\lambda}\right)}\left(\ell^{1}\left(G_{p_{\lambda}}\right)\right): \lambda \in J\right\}
$$

and so, for each $\lambda \in J, \mathbb{M}_{E\left(D_{\lambda}\right)}\left(l^{1}\left(G_{p_{\lambda}}\right)\right)$ is a homomorphic image of $\ell^{1}(S)$. Thus, by [22, Theorem 2.6 (i)], we have

$$
\mathbb{M}_{E\left(D_{\lambda}\right)}\left(\ell^{l}\left(G_{p_{\lambda}}\right)\right) \cong \mathbb{M}_{E\left(D_{\lambda}\right)}(\mathbb{C}) \hat{\otimes}\left(\ell^{1}\left(G_{p_{\lambda}}\right)\right)
$$

is left character amenable for each $\lambda \in J$. Also, $\ell^{1}\left(G_{p_{\lambda}}\right)$ is left character amenable by Corollary 3.3, and so $G_{p_{\lambda}}$ is an amenable group by [22, Corollary 2.4].

Corollary 4.9 Let $S$ be a Brandt semigroup over a group $G$ with index set $J$. Suppose $\ell^{1}(S)$ is left character amenable. Then $G$ is amenable.

Proof It is easy to see that $E(S)=\left\{\left(e_{G}\right)_{i i}: i \in J\right\} \cup\{0\}$. Also, since $E(S)$ is semilattice, we have

$$
(x]=\{y \in E(S): y=y x\}=E(S) x=\left\{\begin{array}{cc}
\{0\} & \text { if } x=0 \\
\{0, x\} & \text { if } x \neq 0
\end{array}\right.
$$

for each $x \in E(S)$. Thus, $(E(S), \leq)$ is uniformly locally finite, and so the result follows by using Theorem 4.8 .

Theorem 4.10 Let $S$ be a Brandt semigroup over a group $G$ with index set $J$. The following are eqivalent:

(i) $\ell^{1}(S)$ is left character amenable

(ii) $G$ is amenable and $I$ is finite

Proof This follows from Proposition 4.4, Proposition 4.6 and Corollary 4.9

Lastly, we give results on Rees Semigroup algebra.

Rees semigroups are described in [14, §3.2] and [8, Chapter 3]. Indeed, let $G$ be a group, and let $m, n \in \mathbb{N}$; the zero adjoined to $G$ is $o$. A Rees semigroup 
has the form $S=\mathcal{M}(G, P, m, n)$; here $P=\left(a_{i j}\right) \in \mathbb{M}_{n, m}(G)$, the collection of $n \times m$ matrices with components in $G$. For $x \in G, i \in \mathbb{N}_{m}$, and $j \in \mathbb{N}_{n}$, let $(x)_{i j}$ be the element of $\mathbb{M}_{m, n}\left(G^{o}\right)$ with $x$ in the $(i, j)^{\text {th. }}$ place and $o$ elsewhere. As a set, $S$ consists of the collection of all these matrices $(x)_{i j}$. Multiplication in $S$ is given by the formula

$$
(x)_{i j}(y)_{k \ell}=\left(x a_{j k} y\right)_{i \ell} \quad\left(x, y \in G, i, k \in \mathbb{N}_{m}, j, \ell \in \mathbb{N}_{n}\right)
$$

it is shown in [14, Lemma 3.2.2] that $S$ is a semigroup.

Similarly, we have the semigroup $\mathcal{M}^{o}(G, P, m, n)$, where the elements of this semigroup are those of $\mathcal{M}(G, P, m, n)$, together with the element $o$, identified with the matrix that has $o$ in each place (so that $o$ is the zero of $\mathcal{M}^{o}(G, P, m, n)$ ), and the components of $P$ are now allowed to belong to $G^{o}$. The matrix $P$ is called the sandwich matrix in each case. The semigroup $\mathcal{M}^{o}(G, P, m, n)$ is a Rees matrix semigroup with a zero over $G$.

We write $\mathcal{M}^{o}(G, P, n)$ for $\mathcal{M}^{o}(G, P, n, n)$ in the case where $m=n$.

The above sandwich matrix $P$ is regular if every row and column contains at least one entry in $G$; the semigroup $\mathcal{M}^{o}(G, P, m, n)$ is regular as a semigroup if and only if the sandwich matrix is regular.

Let $S=\mathcal{M}^{o}(G, P, m, n)$. For $x \in G,(x)_{i j}$ is identified with the element of $\mathbb{M}_{m, n}\left(\ell^{1}(G)\right)$ which has $\delta_{x}$ in the $(i, j)^{\text {th. }}$ position and 0 elsewhere, and $o$ is identified with $\delta_{o}$. Thus an element of $\ell^{1}(S)$ is identified with an element of $\mathbb{M}_{m, n}\left(\ell^{1}(G)\right) \cup \mathbb{C} \delta_{o}$. The sandwich matrix $P \in \mathbb{M}_{n, m}\left(G^{o}\right)$ is identified with a matrix $P \in \mathbb{M}_{n, m}\left(\ell^{1}(G)\right)$ as follows: if the first matrix $P$ has $a \in G$ in the $(i, j)^{\text {th. }}$-position, then the new matrix $P$ has the point mass $\delta_{a}$ in the $(i, j)^{\text {th. }}$ position; if the first matrix $P$ has the element $o$ in the $(i, j)^{\text {th. }}$-position, then the new matrix $P$ has the element $0 \in \ell^{1}(G)$ in the $(i, j)^{\text {th. }}$-position. Thus, as in [8], we can write

$$
\ell^{1}(S)=\mathcal{M}^{o}\left(\ell^{1}(G), P, m, n\right)=\mathcal{M}\left(\ell^{1}(G), P, m, n\right) \oplus \mathbb{C} \delta_{o}
$$

the multiplication is given explicitly in $[8, \mathrm{pp} .61,62] \cdot \ell^{1}(S)$ is called the Rees semigroup algebra. 
It was claimed in [8] that the quotient Banach algebra $\ell^{1}(S) / \mathbb{C} \delta_{o}$ is isometrically isomorphic to the Munn algebra $\mathcal{M}\left(\ell^{1}(G), P, m, n\right)$, where $\mathbb{C} \delta_{o}$ is a one-dimensional ideal. $\ell^{1}(S) / \mathbb{C} \delta_{o}=\mathcal{M}\left(\ell^{1}(G), P, m, n\right)$, is unital. With $m=n$, since $\mathcal{M}\left(\ell^{1}(G), P, n, n\right)=\mathcal{M}\left(\ell^{1}(G), P, n\right)$, is also unital and so the Munn algebra $\mathcal{M}\left(\ell^{1}(G), P, n\right)$, is topologically isometric to $\mathcal{M}_{n}\left(\ell^{1}(G)\right)$, With this identification, we have the next results on Rees semigroup algebra.

Theorem 4.11 Let $S=\mathcal{M}^{o}(G, P, n)$ be a Rees matrix semigroup with a zero over the group $G$ and Sandwich matrix $P$. Then $\ell^{1}(S)$ is left character amenable if and only if it is amenable.

Proof (i) Suppose $\ell^{1}(S)$ is left character amenable. Then $\ell^{1}(S) / \mathbb{C} \delta_{o}$ is left character amenable by Proposition 3.1. Also, since $\ell^{1}(S) / \mathbb{C} \delta_{o}$ is isomorphic to $\mathcal{M}_{n}\left(\ell^{1}(G)\right)$, then $\ell^{1}(S) / \mathbb{C} \delta_{o}$ is amenable by Proposition 3.4. Thus $\ell^{1}(S)$ is amenable by [8, Proposition 2.2 (vi)].

Theorem 4.12 Let $S=\mathcal{M}^{o}(G, P, n)$ be a Rees matrix semigroup with a zero over the group $G$ and Sandwich matrix $P$. Then $\ell^{1}(S)$ is left character amenable if and only if $G$ is amenable.

Proof This follows from the above isometric isomorphism and the fact that the left character amenability of $\mathcal{M}_{n}\left(\ell^{1}(G)\right)$, is equivalent to its amenability see Proposition 3.4 and the hereditary property of amenability in [8, Proposition $2.2(\mathrm{vi})]$

Acknowledgement This work was initiated during the visit of the first author to the African Institute for Mathematical Sciences, (AIMS), South Africa as well as a short visit to the University of Pretoria, South Africa. He gratefully acknowledge the support of AIMS and hospitality of the staffs during the visit. The authors are also grateful to Professor Massoud Amini for bringing paper [2] to their attention. Finally, the authors thank the referee for suggestions which improved the paper, in particular, the remark on Proposition 4.4 and paper [1] that was brought to our attention. 


\section{References}

[1] H. Pourmahmood-Aghababa, L.Y. Shi and Y.J. Wu, Generalized notions of character amenability, Acta Mathematica Sinica, English Series, 29, Issue 7, (2013), 1329 - 1350 .

[2] M. Amini, M. Essmaili, and M. Rostami, Left $\varphi$-biflatness of Banach algebras, preprint.

[3] A. Bodaghi and M. Amimi, Module character amenability of Banach algebras, Arch. Math. (Basel), 99 (2012), 353 - 365.

[4] A.H. Clifford and G.B. Preston, The algebraic theory of semigroups, it Amer. Math. Soc. Surveys 7/I (1961).

[5] E. Essmaili and M. Filali, $\varphi$-Amenability and character amenability of some classes of Banach algebras, Houston J. Math., 39, No 2, (2013), 515 - 529.

[6] M. Bami Lashkarizadeh and H. Samea, Approximate amenability of certain semigroup algebras, it Semigroup Forum 71 (2005), 312 - 322.

[7] H.G. Dales, Banach algebras and automatic continuity, London Mathematical Society Monographs, New Series, Volume 24, The Clarendon Press, Oxford, 2000.

[8] H. G. Dales, A. T.-M. Lau, and D. Strauss, Banach algebras on semigroups and their compactifications, Memoirs Amer. Math. Soc. 205 (2010), 1-165.

[9] J. Duncan and I. Namioka, Amenabilty of inverse semigroup and their semigroup algebras, Proc. Royal Soc. Edinburgh A 80 (1978), 309 - 321.

[10] J. Duncan and A.L.T. Paterson, Amenabilty of discrete convolution semigroup algebras, Math. Scand. 66 (1990), 141-146.

[11] G.H. Esslamdzadeh, Banach algebra structure and amenability of a class of matrix algebras with applications, J. Funct. Anal. 161, (1996), 364 - 383. 
[12] N. Gronbaek, Amenability of weighted discrete semigroup convolution algebras, Proc. R. Soc. Edinb. A 110 (1998), 351 - 360.

[13] A. Ya. Helemskii, Flat Banach module and amenable algebras, Trans. Moscow Math. Soc. 47 (1985), 199-244.

[14] J.M. Howie, Fundamentals of semigroup theory, London Mathematical Society Monographs, Volume 12, The Clarendon Press, Oxford, 1995.

[15] Z. Hu, M.S. Monfared and T. Traynor, On Character amenable Banach algebra, Studia Math. 193 (1) (2009), 53-78.

[16] B. E. Johnson, Cohomology in Banach algebras, Memoirs Amer. Math. Soc. 127 (1972).

[17] E. Kaniuth, A.T. Lau and J. Pym, On $\varphi$-amenability of Banach algebras, Math. Proc. Camb. Phil. Soc. 144 (2008), 85-96.

[18] O.T. Mewomo, On n-weak amenability of Rees semigroup algebras, Proc. Indian Academy of Sci. (Math. Series), Vol. 118 (4) (2008), 547 - 555.

[19] O.T. Mewomo, Various notions of amenability in Banach algebras, Expo. Math. 29 (2011), 283-299.

[20] O.T. Mewomo and O.J. Ogunsola, On n-weak amenability of semigroup algebras, J. Nig. Math. Soc. 32 (2013) 289-301.

[21] O.T. Mewomo and N.B. Okelo, On approximate character amenability of Banach algebras, J. Nig. Math. Soc. 32 (2013), 303-315.

[22] M.S. Monfared, Character amenability of Banach algebras, Math. Proc. Camb. Phil. Soc. 144 (2008), 697-706.

[23] P. Ramsden, Biflatness of semigroup algebras, Semigroup Forum 79 (2009), 515 - 530. 
[24] M.M. Sadr and A. Pourabbas, Approximate amenability of Banach catergory algebras with application to semigroup algebras, Semigroup Forum 79, (2009), 55 - 64 .

[25] A. Sahami and A. Pourabbas, On $\varphi$ - biflat and $\varphi$ - biprojective Banach algebras, Bull. Belegian Math. Soc. Simon Stevin, to appear.

O. T. Mewomo,

Permanent Address:

School of Mathematics, Statistics and Computer Sciences,

University of Kwazulu-Natal, Durban,

South Africa.

Email: mewomoo@ukzn.ac.za

Temporary Address:

African Institute for Mathematical Sciences,

Muizenberg, Cape Town, South Africa.

Email:tosin@aims.ac.za

S.M. Maepa,

Department of Mathematics and applied Mathematics,

University of Pretoria,

Pretoria, South Africa.

Emaill:charles.maepa@up.ac.za 